\title{
Geschichte der Dermatologie: Der Dessauer Hautarzt Dr. med. Klaus Holzegel
}

\author{
History of Dermatology: The Dermatologist Dr. Klaus Holzegel from Dessau
}

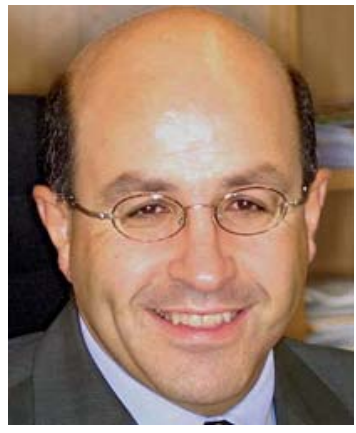

Prof. Dr. med.

Christos C. Zouboulis

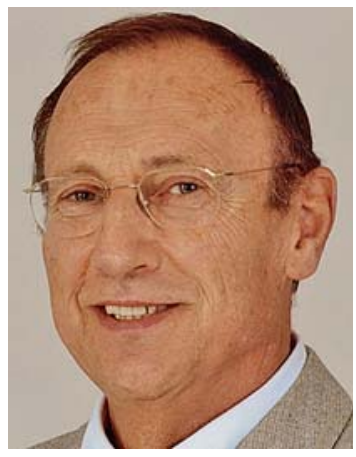

Prof. Dr. med.

Hans-Dieter Göring

\section{Bibliografie}

Dol http://dx.doi.org/ 10.1055/s-0029-1215345 Online-Publikation: 1. 12. 2009 Akt Dermatol 2010; 36; 1-2 (c) Georg Thieme Verlag KG . Stuttgart · New York ISSN 0340-2541

\section{Korrespondenzadresse}

Prof. Dr. Christos C. Zouboulis Klinik für Dermatologie, Venerologie und Allergologie/ Immunologisches Zentrum, Städtisches Klinikum Dessau Auenweg 38 06847 Dessau-Roßlau christos.zouboulis@ klinikum-dessau.de
Dieses Heft der Aktuellen Dermatologie wird der Geschichte der Dermatologie und Herrn Dr. med. Klaus Holzegel, Hautarzt aus Dessau, gewidmet. Den Herren Professoren Karl Holubar, Medizinische Universität Wien, und Albrecht Scholz, Medizinische Fakultät Carl Gustav Carus der Technischen Universität Dresden, sowie Herrn Dr. Christoph Löser, Hautklinik des Klinikums Ludwigshafen, gilt unser außerordentlicher Dank für ihre wertvollen Beiträge.

Für manche jüngere Kollegen kann es zu der Frage kommen: Wer ist Dr. Holzegel und warum wird er geehrt? Tatsächlich hat Dr. Holzegel Hervorragendes auf den Gebieten der Phlebologie, der wissenschaftlichen ärztlichen Fortbildung und der ärztlichen Berufspolitik geleistet, aber er ist vor allem ein Pionier der Wiedervereinigung der Dermatologie in Deutschland.

1965 begann Dr. Holzegel als Leiter der Hautabteilung der Betriebspoliklinik und des Betriebskrankenhauses Wolfen, Sachsen-Anhalt, aus eigenem Impetus die Subspezialität Phlebologie aufund auszubauen. Er gehört zu den Pionieren dieses Fachgebietes. Er verfasste zahlreiche Veröffentlichungen, unter anderem wichtigen Beiträge zur Diagnostik und Therapie der chronisch-venösen Insuffizienz, ihrer Folgeerkrankungen und Komplikationen.

Für diese Krankheit, die für eine große Anzahl der Betroffenen einen hohen Leidensdruck besaß und deren sozialmedizinische Dimension groß ist, fehlte anfänglich bei den staatlichen Leitungshierarchien der DDR das erforderliche Problembewusstsein. Tausende hilfesuchender Patienten erfuhren durch Dr. Holzegel wirkungsvolle Hilfe, die z.T. von anderen Krankenhäusern, Polikliniken, einschließlich mancher Universitätskliniken, nicht geboten werden konnte.

Während seiner 11-jährigen Wolfener Tätigkeit haben Kollegen aus der gesamten DDR bei ihm hospitiert und spezielle Kenntnisse auf dem Gebiet der Phlebologie erworben. Außerdem hat Holzegels rege Publikations- und Vortragstätigkeit dazu beigetragen, dass trotz systemimmanenter Defizite die ostdeutsche Phlebologie ein recht passables Niveau erreichte. Dies geht auch aus dem gemeinsam mit Dr. Oswald Petter, Torgau, verfassten Buch „Zur Geschichte der deutschen Phlebologie“ hervor.

Aufgrund zunehmender und erheblicher Behinderung seiner Publikationstätigkeit, speziell auf dem Gebiet der Berufsdermatosen, regelmäßiger Beschlagnahme von Fachzeitschriften durch den DDR-Zoll und deswegen bestehender Zerwürfnisse mit der Verwaltungsleitung flüchtete Holzegel
1976 nach Niedersachsen, setzte in eigener Praxis in Wunstorf seine ärztliche und wissenschaftliche Arbeit fort und gründete mit Gleichgesinnten den Berufsverband der Phlebologen, eine, wie sich zeigte, erfolgreiche interdisziplinäre Vereinigung. Über viele Jahre trug Holzegel wesentlich zur Pflege internationaler Kontakte zu den ausländischen Phlebologen, besonders Österreichs und der Schweiz, bei.

Von den 255 Publikationen Dr. Holzegels und seinen zahlreichen Vorträgen befasst sich ein gutes Drittel mit phlebologischen Problemen. Viele Erkenntnisse aus seiner eigenen wissenschaftlichen Tätigkeit flossen in die Fortbildung ein. Sie zeigen beispielhaft, dass man auch als niedergelassener Arzt erfolgreich wissenschaftlich arbeiten kann. Davon zeugt auch seine Tätigkeit als Hauptschriftleiter der Monatszeitschrift „Der Deutsche Dermatologe“ von 1992-2000. Ihm gelang es, diese Verbandszeitschrift zu einem anspruchsvollen Journal zu profilieren.

Bis heute ist Dr. Holzegel Mitglied im wissenschaftlichen Beirat der Zeitschrift „DERM“ und berichtet regelmäßig über die Alfred-Marchionini-Tagungen in Hamburg und die Herbsttagungen „Fortschritte der Allergologie, Dermatologie, Pneumologie und Immunologie“ in Davos. Berufspolitisch war Dr. Holzegel von 1979-1985 Schatzmeister des Berufsverbandes der Deutschen Dermatologen (BVDD), von 1980-1989 war er Vorsitzender des Landesverbandes Niedersachsen.

Besondere Verdienste erwarb sich Dr. Klaus Holzegel beim Aufbau der neuen Strukturen der ambulanten Tätigkeit in den neuen Bundesländern. Als „Sonderreferent für DDR-Verbindungen“ im BVDD stand er vielen Dermatologen mit Rat und Tat zur Seite und half beim Aufbau der neuen Landesverbände, an deren Gründung er teilnahm (Sachsen: 15. 6. 1990 in Dresden, Thüringen: 7.7.) 29. 9. 1990 in Erfurt, Sachsen-Anhalt: 26.9.1990 in Halle, Berlin [Ost]: 17.10.1990 in der Charité, Mecklenburg-Vorpommern: 17.11.1990 in Rostock, Brandenburg: 9.12.1990 in Potsdam). Besonderer Tätigkeitsschwerpunkt bildete hier sein Landesverband Sachsen-Anhalt, der ihm ebenso wie Niedersachsen die Ehrenmitgliedschaft verlieh.

Mehrere Ehrungen sind Dr. Holzegel zuteil geworden: Karl-Linser-Medaille (1990), Verdienstmedaille des BVDD (1991), Bismarck-Medaille in Silber (1992), Ernst-von-Bergmann-Plakette (1999) und Bundesverdienstkreuz am Bande (1997).

Seit 10 Jahren ist Dr. Holzegel Vorsitzender des Ehrenrates des BVDD. Seinem diplomatisch aus- 
gleichenden Geschick ist es zu danken, dass es bereits im Vorfeld von Ehrengerichtsverfahren meist zu einer einvernehmlichen Regelung kommt.

Auch im Ruhestand ist Herr Dr. Holzegel bis zum heutigen Tag wissenschaftlich tätig. Er kehrte nach Dessau zurück, wo er wieder seinen ständigen Wohnsitz nahm, und beschäftigt sich seit Jahren mit der Geschichte der Dermatologie. So erschien 2007 eine viel beachtete Veröffentlichung über die praktisch in Vergessenheit geratene Entwicklung eines Neo-Salvarsan-Analogons in Magdeburg durch den Chemiker Prof. Dr. Schmitz, dessen Großproduktion bei Fahlberg-List ermöglichte, die bedrohlich hohe Zahl von Luesinfektionen in Deutschland nach dem 2. Weltkrieg erfolgreich zu behandeln [1]. Insbesondere widmete er sich der Erforschung der Dessauer Dermatologie [2], welche ihm ihre genaue Darstellung im Jubiläumsbuch „Geschichte der deutschsprachigen Dermatologie“ (Hrsg.: Scholz A, Holubar K, Burg G, Gollnick H, 2009) verdankt. Ohne seine Bemühungen wäre wahrscheinlich die Darstellung der Geschichte der Dessauer Hautklinik, wie die vieler anderer Hautkliniken aus den neuen Bundesländern, viel kürzer und ungenauer geblieben.

Herr Dr. med. Klaus Holzegel wird anlässlich seines 75. Geburtstages am 22. November 2009 aufgrund seiner herausragenden Leistungen und bleibenden Verdienste mit der Silbernen Ehrennadel der Ärztekammer Sachsen-Anhalt geehrt.

Prof. Dr. med. Christos C. Zouboulis Chefarzt der Klinik für Dermatologie, Venerologie und Allergologie/Immunologisches Zentrum

Prof. Dr. med. Hans-Dieter Göring Vorsitzender des Tumorzentrums Anhalt

\section{Literatur}

1 Holzegel K, Göring H-D. „Ein Glücksfall für die Ostzone...“ - Die Synthese des Neo-Salvarsan-Analogons Arsaminol (Neo-Arsoluin) durch Prof. Dr. Ernst Schmitz und dessen Bedeutung für die Eindämmung der Syphilis in der Sowjetischen Besatzungszone Deutschlands. Akt Dermatol 2007; 33: $485-487$

2 Holzegel K. Zur Geschichte der Dermatologie Dessaus und seiner Hautklinik. Akt Dermatol 2006; 32: 285-289
Herrn Prof. Holzegel gewidmet ist ebenfalls die Arbeit „Dem Leiden war er, war dem Tod vertraut" Friedrich Schiller aus Anlass seines 250. Geburtstages - Der Einfluss von Krankheit und Leiden auf sein Leben und Schaffen von H.-D. Göring, die bereits in Heft 11/2009 der Aktuellen Dermatologie erschienen ist. 